\title{
Key milestones of infographics evolution in cartography
}

\author{
Jakub Konicek ${ }^{\mathrm{a}, *}$ \\ ${ }^{a}$ Department of Geoinformatics, Palacký University Olomouc, 17. Listopadu 50, 77900 Olomouc, Jakub Konicek - \\ jakub.konicek@upol.cz
}

* Corresponding author

Keywords: infographics, cartography, milestones, evolution

\begin{abstract}
:
Infographic is a popular term which resonates quite significantly in the world of the Internet for a long time. It became a very often used phrase not only in various forums, blogs, and websites, but also turned into a part of the scientific research presentation and later as a field of study in (not only) cartographic visualization as well. Either, the cartography is no exception. Many cartographers practically used this term and its generally known principles. Nevertheless, principles and its well-known essence are not officially and uniformly defined yet.
\end{abstract}

The paper aims to present the evolution of infographics in time and its intersection and the usage focusing on cartography. The main aim is to figure out trends and milestones which are noticeable from the gradual evolution of infographics. The interests and activities of the professional scientific groups of the International Cartographic Association, as well as the thematic focus of the papers at the annual international conferences, confirm the growing popularity of the professional public.

The case study figures out, through practical examples, when infographic has become a valuable part of cartographic creation and subject of study in cartographic research. Based on the available literature, professionally oriented forums and thematically focused articles at the Web of Science, the paper seeks to show an extended view of infographics. According to trendsetters, it is a popular design concept, nevertheless, it is still not given significant scientific attention from a professional point of view.

The motivation for this research is to compile an evolution timeline of infographics, describe significant trends and define key milestones influencing its development, especially in the field of practical usage in cartography. 